\title{
Assessing the utility of Genexpert as a diagnostic tool in the detection of Tuberculosis in a neurological tertiary care setup
}

\author{
Sourav Maiti, Pearl A. Prabal ${ }^{\star}$ \\ Institute of Neurosciences, Kolkata, India \\ Correspondence E-mail : smaiti76@gmail.com; pearlprabal@gmail.com*
}

\begin{abstract}
Tuberculosis in the CNS(central nervous system) is the most dangerous form of extrapulmonary Tuberculosis and is still a formidable clinical challenge. Rapid diagnosis of Tuberculosis and detection of Rifampicin resistance are essential for early disease management. The Xpert/Rif Assay is an automated nucleic acid amplification test that can identify the Mycobacterium tuberculosis DNA and resistance to Rifampicin. In this experiment we used different pulmonary as well as extrapulmonary samples from patients to assess the utility of genexpert as a diagnostic tool.
\end{abstract}

Keywords: Genexpert, Tuberculosis, central nervous system, Xpert MTB/Rif assay

\section{Introduction}

Tuberculosis is an infectious disease that usually affects the lungs and can spread to other parts of the body. It is the second biggest killer globally. Diagnosis of Tuberculosis involves screening of the lungs and lymph nodes, blood tests, chest x-rays, sputum tests and other molecular methods that are used for detection. Symptoms and medical history are very important to assess the individual's risk of exposure to Tuberculosis. Multi drug resistant Tuberculosis is more difficult to diagnose.

Genexpert is a relatively new test for Tuberculosis. It has been declared by WHO as the gold standard for the detection of Tuberculosis from various samples. The Xpert $\mathrm{MTb} /$ Rif assay detects DNA sequences specific for Mycobacterium tuberculosis and Rifampicin resistance by the Polymerase chain reaction. It is a rapid and easy to use Nucleic acid amplification test.

Different manifestations of Tuberculosis include:

\section{Cranial Tuberculosis:}

Haematogenous spread from the lungs or gastrointestinal tract is most common, leading to small subpial or subependymal infective foci. These are termed Rich foci and form a reservoir from which intracranial manifestations may arise. This can occur either during the primary infection (uncommon, and more frequently seen in children) or be reactivated later and cause a post-primary infection.(R Bryan.et al,2008)There is a wide radiological and pathological spectrum of the CNS disease. Tuberculous meningitis and intracranial tuberculous granuloma (tuberculoma) are the most common manifestations. Treatment of CNS tuberculosis is based on an anti-tubercular treatment regimen. However, multidrug-resistant tuberculosis remains a major hurdle in treatment. 
Spinal Tuberculosis :

Spinal tuberculosis (Cherian \& Thomas, 2011) is a destructive form of tuberculosis. It accounts for approximately half of all cases of musculoskeletal tuberculosis. Spinal tuberculosis is more common in children and young adults. The incidence of spinal tuberculosis is increasing in developed nations. Genetic susceptibility to spinal tuberculosis has recently been demonstrated. Characteristically, there is destruction of the intervertebral disk space and the adjacent vertebral bodies, collapse of the spinal elements, and anterior wedging leading to kyphosis and gibbus formation. The thoracic region of vertebral column is most frequently affected. Formation of a 'cold' abscess around the lesion is another characteristic feature. The incidence of multi-level non contiguous vertebral tuberculosis occurs more frequently than previously recognized. Common clinical manifestations include constitutional symptoms, back pain, spinal tenderness, paraplegia, and spinal deformities. For the diagnosis of spinal tuberculosis magnetic resonance imaging is more sensitive imaging technique than $x$-ray and more specific than computed tomography. Magnetic resonance imaging frequently demonstrates involvement of the vertebral bodies on either side of the disk, disk destruction, cold abscess, vertebral collapse, and presence of vertebral column deformities. Neuroimaging-guided needle biopsy from the affected site in the centre of the vertebral body is the gold standard technique for early histopathological diagnosis. Anti TB treatment remains the cornerstone of treatment. Surgery may be required in selected cases, e.g. large abscess formation, severe kyphosis, an evolving neurological deficit, or lack of response to medical treatment. With early diagnosis and early treatment, prognosis is generally good.

\section{Lymph node Tuberculosis :}

Tuberculous lymphadenitis usually presents as a gradually increasing painless swelling of one or more lymph nodes of weeks to months duration. Some patients, especially those with extensive disease or a co-existing disease, may have systemic symptoms i.e. fever, weight loss, fatigue and night sweats.
Distressing cough may be a prominent symptom in mediastinal lymphadenitis. Initially the nodes are firm, discrete and mobile. The overlying skin is free. Later, the nodes may become matted and the overlying skin inflamed. In more advanced stage, the nodes may soften leading to formation of abscesses and sinus tracts which may be difficult to heal. Unusually large nodes may compress or invade the adjoining structures complicating the course of the disease (Gupta, 2004).

\section{Materials and Methods}

Sample preparation and History Pulmonary samples as well as extrapulmonary samples are used for this study.

History of illness gathered from individual patient files from their respective wards, details regarding past history of illness at the time of admission, previous episodes, signs and symptoms, procedures performed(if any), MRI and CT reports as well as medications the patient is on is checked and noted with patient's consent.

\section{Gram staining}

Gram staining is a common technique used to differentiate two large groups of bacteria based on their different cell wall constituents. The Gram stain procedure distinguishes between Gram positive and Gram negative groups by colouring these cells red or violet.

\section{Staining:}

Place slide with heat fixed smear on staining tray. Gently flood smear with crystal violet and let stand for 1 minute. Tilt the slide slightly and gently rinse with tap water or distilled water using a wash bottle. Gently flood the smear with Gram's iodine and let stand for 1 minute. Tilt the slide slightly and gently rinse with tap water or distilled water using a wash bottle. The smear will appear as a purple circle on the slide. Decolorize using $95 \%$ ethyl alcohol or acetone. Tilt the slide slightly and apply the alcohol drop by drop for 5 to 10 seconds until the alcohol runs almost clear. Be careful not to over-decolorize. Immediately rinse with water. Gently flood with Safranin to counter-stain and let stand for 45 seconds. Tilt the slide slightly and gently rinse with tap water or distilled 
water using a wash bottle. Blot dry the slide with bibulous paper. View the smear using a light-microscope under oil-immersion.

\section{Ziehl Neelsen staining}

Ziehl-Neelsen (ZN) method of Acid Fast staining technique is used to stain Mycobacterium species including $M$. tuberculosis, M. ulcerans, and M. leprae and non-tuberculous mycobacteria (NTM). Detection of acid-fast bacilli (AFB) in stained and acid-washed smears examined microscopically may provide the initial bacteriologic evidence of the presence of mycobacteria in a clinical specimen. Smear microscopy is the quickest and easiest procedure that can be performed. The cell wall of Mycobacteria contain high concentration of lipid making them waxy, hydrophobic, and impermeable to routine stain such as the Gram Stain.

\section{Method :}

- Spread the sputum evenly over the central area of the slide using a continuous rotational movement. The recommended size of the smear is about $20 \mathrm{~mm}$ by $10 \mathrm{~mm}$.

- Place slides on dryer with smeared surface upwards, and air dry for about 30 minutes.

- Heat fix dried smear.

- Cover the smear will Carbol fuschin stain

- Heat the smear until vapour just begins to rise (i.e. about 60 degree Celsius). Do not overheat (boil or dry). Add additional stain if necessary. Allow the heated stain to remain on the slide for 5 minutes.

- Wash off the stain with clean water.

- Cover the smear with $20 \%$ sulfuric acid until the smear is sufficiently decolorized, i.e. pale pink.

- Wash well with clean water

- Cover the stain with malachite green stain for 1-2 minutes

- Wash off stain with clean water

- Examine the smear microscopically, using the $100 x$ oil immersion objective (10X eye piece for a total of 1000X magnification) and scan the smear systematically.

\section{Samples run on GeneXpert}

After samples are segregated during sample preparation, they are further processed for the automated detection of Mycobacterial complex as well as Rifampicin resistance via the GeneXpert. The test is a molecular test based on nested real-time polymerase chain reaction that can give a result in less than 2 hours. The sample is treated with the provided buffer, shaken and set aside for ten minutes initially then shaken again and kept aside for another five minutes to ensure complete digestion of any residual material by the buffer solution.

The resultant solution is then drawn with the provided dropper and added to the cartridge which has been pre labelled. This cartridge is then loaded onto the Cepheid automated genexpert system IV and after entering all the relevant patient details each cartridge is scanned via their unique identification bar code and then run on the machine.
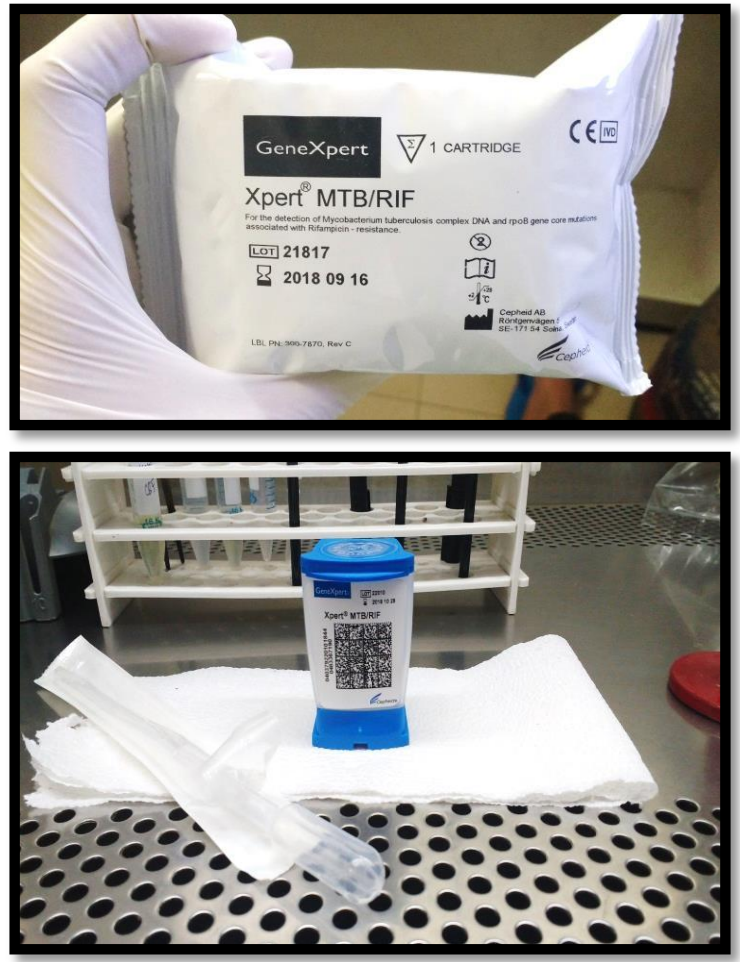
Interpretation of results and clinical
correlation

After the sample is run the machine gives us negative or positive results accordingly along 
with details of Rifampicin resistance or sensitivity. The load of the organism is also detected as very low, low, medium and high. The results are then clinically correlated with radiological scans, symptoms, biochemistry analysis, routine culture, staining as well as mycobacterial culture which takes about 6 weeks.

\section{Exclusion Principle}

- Sample state - Unacceptable samples like grossly bloody samples

- Sample volume - Inadequate sample volumes

\section{Inclusion Principle}

- Samples submitted for testing for Tuberculosis

\section{Ethical Approval}

Ethical Approval was obtained from the Ethical Committee Review Board at the Institute of Neurosciences, Kolkata. Patient approval regarding the use of their medical records and information; was obtained.

\section{Results and Discussion}

A total of 248 samples were analysed for this study. Our data was subdivided into different categories based on the type of samples received at the hospital into cranial, spinal and lymph node samples.

Table : CRANIAL SAMPLES: A total of 182 samples were obtained.

\begin{tabular}{|l|l|l|}
\hline Cranial samples & Positive & $\%$ Positive \\
\hline $\mathbf{1 8 2} / \mathbf{2 4 8}$ & 24 & $13.81 \%$ \\
\hline Total $\%=\mathbf{7 3 . 3 8} \%$ & & \\
\hline
\end{tabular}

Samples were screened for Tuberculosis meningitis with or without Hydrocephalus as well as the presence of tumours, among which the number of malignancies were noted.

Table: Samples Processed

\begin{tabular}{|c|c|c|c|}
\hline TBM/Hydrocephalus(+or-) & Genexpert (+) & \% Positive & \\
\hline $\mathbf{1 4}$ & 5 & $35.71 \%$ & \\
\hline Brain tumour/ brain lesions & Genexpert (+) & Culture & Other \\
\hline $\mathbf{1 6}$ & 2 & 0 & 12 (5 Malignancies) \\
\hline \% Positive & $12.5 \%$ & & \\
\hline
\end{tabular}

Table : $L Y M P H$ NODE SAMPLES: A total of 2 samples were obtained

\begin{tabular}{|c|c|c|c|}
\hline Lymph node samples & Genexpert (+) & Culture & $\%$ Positive \\
\hline $\mathbf{2} / \mathbf{2 4 8}$ & 2 & 1 & $100 \%$ \\
\hline Total $\%=\mathbf{0 . 8 0} \%$ & & & \\
\hline
\end{tabular}

Table : SPINAL SAMPLES : A total of 24 samples were obtained

\begin{tabular}{|c|c|c|c|c|}
\hline Spinal samples & Genexpert (+) & Culture & Others & ZN \\
\hline $\mathbf{2 4 / 2 4 8}$ & 6 & 5 & 7 & 2 \\
\hline Total \%= 9.67\% & & & & \\
\hline Total Positive $=\mathbf{1 1}$ & & & & \\
\hline$\%$ positive $=\mathbf{4 5 . 8 3 \%}$ & & & & \\
\hline
\end{tabular}




\section{Spinal sample screening}

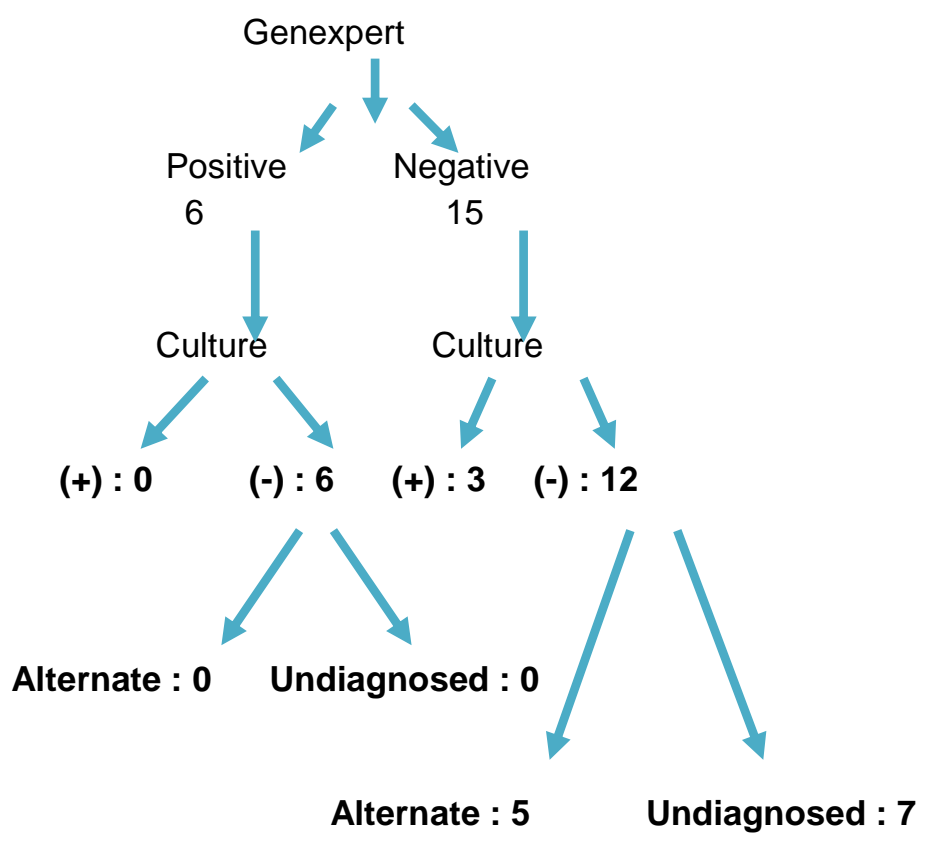

In the case of cranial samples( mostly tissue and CSF), suspected samples with increased or decreased suspicion for Tuberculosis, the definitive diagnosis of Tuberculosis was found to be positive for $13.81 \%$ of the cases during the period this study was conducted. Cranial Tb samples constituted $73.38 \%$ of the total number of samples used here which is expected in a neurospecialty setup. In order to understand the clinical aspect of the diagnosis we also studied the number of TB meningitis cases in patients with or without hydrocephalus where we found that 5 out of 14 cases were found positive for tuberculosis giving us a positive $\%$ of $35.71 \%$. We also studied the presence of brain tumours and looked into the cases with malignancies in order to exclude $\mathrm{Tb}$ and to get a clear picture of the diagnosis radiologically. Out of 16 cases of brain tumours/lesions 2 were positive for genexpert, none of the samples showed a positive result for culture, 12 of the cases showed an alternative diagnosis with 5 out of these 12 positive for malignancies. Centrifuged cerebrospinal fluid samples were found to provide a better yield.

In the case of lymph node samples (mostly pus and lymphadenopathy samples), two samples were obtained, both were positive for Genexpert and one showed positive results in culture, giving us a $100 \%$ positive percentage. These samples constituted $0.80 \%$ of the total number of samples used in this study.

In the case of spinal samples (mostly FNA material, psoas abscess, paravertebral fluids) out of 24 samples, 6 were positive for genexpert, 5 for culture, 2 for Ziehl Neelsen staining, 7 others showed alternative diagnosis giving us a positive percentage of $45.83 \%$. Alternative diagnosis included degenerative changes in the spine or Spondilysis. These samples constituted $9.67 \%$ of the total number of samples used in this study.

Detection of the bacteria is seen to be highest in the case of Lymph node and Spinal samples as the load of bacteria is generally higher in these samples as compared to the cranial samples like cerebrospinal fluid. The sample size used for these categories of samples is also less, in the case of different tissue samples used, Tb Bacilli usually reside in central caseation of the tissue, where the bacterial DNA may also be found without the bacteria itself. In many cases, the peripheral tissues may've been used as samples for diagnosis (as sent by physicians to the laboratory) in this case we might not get a clear picture in terms of the disease and bacterial load. 
Table : Culture Results

\begin{tabular}{|l|c|c|c|}
\hline & & Genexpert & $\%$ positive \\
\hline Culture (+) & 6 & 3 & $50 \%$ \\
\hline Culture (-) & 18 & 5 & $27.77 \%$ \\
\hline
\end{tabular}

Table : Different Natures of Samples used

\begin{tabular}{|l|c|c|c|}
\hline Sample nature & Number of samples & Genexpert (+) & \% Positive \\
\hline pulmonary & 16 & 6 & $37.5 \%$ \\
\hline extrapulmonary & 232 & 24 & $10.34 \%$ \\
\hline
\end{tabular}

In terms of culture reports, 3/6 showed positive for both culture and Genexpert as well, giving us a $50 \%$ positive correlation between the two diagnostic techniques. A previous study found that the MTB/RIF assay had a calculated limit of detection of $131 \mathrm{CFU} / \mathrm{ml}$ of sputum and was able to detect as few as $10 \mathrm{CFU} / \mathrm{ml}$ of sputum in $35 \%$ of samples (Helb et al. 2010). In the study, the longer turnaround time for the MTB/RIF test-negative samples could be due to low numbers of bacilli which were under the limits of detection of the test. While the limit of detection for culture in liquid media is $10-50$ colony forming units $/ \mathrm{ml}$, samples must contain about 10,000 colony forming units/ml to be positive on smear microscopy (Lawn, et al. 2013) which could explain why cultures can sometimes provide false negative results. On the other hand, some samples may also have a bacillary load lower than $131 \mathrm{cfu} / \mathrm{ml}$ and this may allow them to go undetected by the Genexpert system of detection as well.

Overall, out of 40 clinical Tuberculosis cases we found 27 gave us positive results via Genexpert. Out of 16 pulmonary samples 6 were detected positive and out of 232 extrapulmonary samples 24 were detected positive. This shows us a higher detection rate for the pulmonary samples as compared to the extrapulmonary ones, this is mainly because pulmonary samples usually carry a higher load of the $\mathrm{Tb}$ bacilli and hence have higher chances of getting detected and lower chances of giving false negatives.

Diagnosing multi-drug resistant and extensively drug-resistant as well as HIVassociated TB can be complex and expensive. In 2016, 4 new diagnostic tests were recommended by WHO - a rapid molecular test to detect TB at peripheral health centres where Xpert MTB/RIF cannot be used, and 3 tests to detect resistance to first- and secondline TB medicines. Tuberculosis is particularly difficult to diagnose in children and as yet only the Xpert MTB/RIF assay is generally available to assist with the diagnosis of pediatric TB (WHO 2016). Rapid and effective diagnosis of patients suspected of having TB remains a challenge. The conventional TB diagnosis techniques, including methods based on direct microscopic examination by ZiehINeelsen staining, culture, chest radiography and tuberculin skin testing (Wallis, et al. 2010) have limitations and are thus not always helpful in diagnosing TB. Smear microscopy alone, although cheap and easy to perform, has a highly false-negative result and cannot identify drug-resistance (Behr, et al. 1999) Currently, only $28 \%$ of expected incident cases of tuberculosis are detected and reported as smear positive (WHO 2010).

Although the culture is more sensitive than the smear microscopy, culture generally provides results in at least 2-8 weeks (Pai et al. 2006) requires biosafety measures, and needs specialized laboratory personnel (WHO, 2010). Cultures can also give positive results in smear negative cases owing to large volumes of samples and provided that the bacilli are still alive. This leads to a diagnostic delay that impedes disease control, and increases healthcare costs. Since the discovery of the polymerase chain reaction (PCR), a large number of molecular techniques have been developed (Cheng, et al. 2005). However, their sensitivity is greatly dependent on the efficiency of the sample preparation, DNA extraction and the presence of PCR inhibitors.

One of latest assay, Xpert MTB/RIF (Cepheid, Sunnyvale, CA, USA) assay. The assay 
detects Mycobacterium tuberculosis (Mtb) and RIF-resistance by PCR amplifying five overlapping probes complementary to the rifampin resistance-determining region (RRDR) of the Mtb rpo B gene, and subsequently probes this region for mutations that are associated with RIF-resistance (Blakemore, et al. 2010).

The PCR amplification process is hemi-nested to minimize cross amplification of nontuberculosis mycobacterium (NTM) species, and to maximize mutation detection (Helb, et al. 2010). RIF-resistance can serve as a marker for MDR-TB. Therefore, the assay may fulfil the requirement of diagnosing TB and MDR-TB. In addition, the assay can diagnose TB with a nearly fully automated manner, including bacterial lysis, nucleic acid extraction and amplification, and amplicon detection. Furthermore, the assay requires minimal biosafety measures (Banada, et al. 2010). A key advantage of Xpert MTB/RIF assay over smear microscopy is the simultaneous assessment to RIF resistance. The falsenegative result may attribute to the analytical limit of detection of Xpert MTB/RIF assay, which was reported to be $131 \mathrm{CFU} / \mathrm{ml}$ of specimen (Blakemore, et al. 2010) However, the culture can detect very low concentrations of organisms e as low as 10 to $100 \mathrm{CFU} / \mathrm{ml}$. The assay can detect RIF-resistance by only probing the rpoB gene, and the mutation points in approximately 5\% RIF-resistant Mtb

\section{References}

Banada, P. P, Sivasubramani, S. K., Blakemore, R., Boehme, C., Perkins, M.D., Fennelly, K., Alland, D. (2010) Containment of bioaerosol infection risk by the Xpert MTB/RIF assay and its applicability to point-of-care settings. J Clin Microbiol. 48(10), 3551-7.

Behr, M. A., Warren, S. A., Salamon H, Hopewell, P. C., Ponce de Leon, A., Daley, C. L., Small, P. M. (1999). Transmission of Mycobacterium tuberculosis from patients smear-negative for acidfast bacilli. Lancet. 6, 353(9151), 444-9.

Blakemore, R., Story, E., Helb, D., Kop, J., Banada, P, Owens, M. R., Chakravorty, S., Jones, M., Alland, D. (2010). Evaluation of the analytical performance of the Xpert MTB/RIF assay. J Clin Microbiol. 48(7), 2495-501. isolates occur outside core rpo $\mathrm{B}$ gene region (Traore, et al. 2000), so it would not be identified by Xpert MTB/RIF assay.

\section{Conclusion}

The scarcity of literature regarding the extra pulmonary diagnosis of Tuberculosis particularly from CSF has furthered the basis of this study. The main objective of this study was to find out the efficiency of this test in the detection of Tuberculosis in the brain. In cases where patients have tuberculomas, the diagnosis chiefly relied on its histopathology which is primarily determined by host responses whereas, identification of the bacteria provides direct proof of its pathogenesis. Direct methods of identification like culture and staining have not proved to be too reliable in terms of diagnosis, hence we explored the efficiency of the GeneXpert system via this study. In conclusion, the MTB/RIF test is less dependent on the user's skills, and routine staff with minimal training can use the test. It has a short turnaround time and simultaneously detects $M$. tuberculosis and RIF resistance in less than 3 hours. Although the MTB/RIF test could be a useful tool for rapid identification of RIF-resistant M. tuberculosis, especially in smear-positive clinical samples, the test results must always be confirmed by culture and DST.

Bryan, R., Michael Olin, Cristina A. Baker, Thomas, Molitor W., Phillip K. Peterson (2008). Central Nervous System Tuberculosis: Pathogenesis and Clinical Aspects Clin. Microbiol. Rev. 21(2), 243-261

Cheng, V. C., Yew, W. W., Yuen, K. Y. (2005). Molecular diagnostics in tuberculosis. Eur $J$ Clin Microbiol Infect Dis, 24(11), 711-20.

Cherian, A., Thomas, S. V. (2011) Central Nervous system. Afri health Sci, 11(1), 116-127.

Gupta, P. R. (2004). Difficulties in managing lymph node tuberculosis. Lung India; 21, 50-3.

Helb, D., et al. (2010). Rapid detection of Mycobacterium tuberculosis and rifampin resistance by use of on-demand, near-patient technology. J. Clin. Microbiol. 48, 229-237 
Helb, D., Jones, M., Story, E., Boehme, C., Wallace, E., Ho, K., Kop, J., Owens, M. R., Rodgers, R., Banada, P., Safi, H., Blakemore, R., Lan, N. T., Jones-López, E. C., Levi, M., Burday, M., Ayakaka, I., Mugerwa, R. D., McMillan, B., Winn-Deen, E., Christel, L., Dailey, P., Perkins, M. D., Persing, D. H., Alland, D. (2010). Rapid detection of Mycobacterium tuberculosis and rifampin resistance by use of on-demand, nearpatient technology. J Clin Microbiol. 48(1), 229-37.

Lawn, S. D., Mwaba, P., Bates, M., Piatek, A., Alexander, H., et al. (2013). Advances in tuberculosis diagnostics: the Xpert MTB/RIF assay and future prospects for a point-of-care test. Lancet Infect Dis 13, 349-361.

Pai, M, Kalantri, S., Dheda, K.. (2006). New tools and emerging technologies for the diagnosis of tuberculosis: part I. Latent tuberculosis. Expert Rev Mol Diagn. 6(3), 413-22.
Traore, H., Fissette, K., Bastian, I., Devleeschouwer, M., Portaels, F. (2000) Detection of rifampicin resistance in Mycobacterium tuberculosis isolates from diverse countries by a commercial line probe assay as an initial indicator of multidrug resistance. Int $J$ Tuberc Lung Dis. 4(5), 481-4.

Wallis, R. S., Pai, M., Menzies, D., Doherty, T. M., Walzl, G., Perkins, M. D., Zumla, A. (2010). Biomarkers and diagnostics for tuberculosis: progress, needs, and translation into practice. Lancet. 29, 375(9729),1920-37.

WHO Factsheet, (2016). available at www.who.int/tb/publications/factsheet_global.pdf

World Health Organization (2009). Global tuberculosis control. A short update to the 2009 report. Geneva, Switzerland.

World Health Organization.Global tuberculosis control (2010). Publication no. WHO/HTM/2010.7. Geneva, Switzerland. 